\title{
A simulation study of short channel effects with a QET model based on Fermi-Dirac statistics and nonparabolicity for high-mobility MOSFETs
}

\author{
Shohiro Sho ${ }^{1} \cdot$ Shinji Odanaka $^{1} \cdot$ Akira Hiroki $^{2}$
}

Published online: 7 October 2015

(C) The Author(s) 2015. This article is published with open access at Springerlink.com

\begin{abstract}
In this paper, the quantum confinement and short channel effects of $\mathrm{Si}, \mathrm{Ge}$, and $\mathrm{In}_{0.53} \mathrm{Ga}_{0.47} \mathrm{As}$ n-MOSFETs are evaluated. Both bulk and double-gate structures are simulated using a quantum energy transport model based on Fermi-Dirac statistics. Nonparabolic band effects are further considered. The QET model allows us to simulate carrier transport including quantum confinement and hot carrier effects. The charge control by the gate is reduced in the Ge and $\mathrm{In}_{0.53} \mathrm{Ga}_{0.47}$ As bulk n-MOSFETs due to the low effective mass and high permittivity. This charge control reduction induces the degradation of short channel effects. In double-gate structures, different improvements of drain induced barrier lowering (DIBL) and subthreshold slope (SS) are seen. The double-gate structure is effective in the suppression of DIBL for all channel materials. The SS degradation depends on channel materials even in double-gate structure.
\end{abstract}

Keywords Quantum energy transport (QET) model · Nonparabolic band effects · Fermi-Dirac statistics · Quantum confinement $\cdot$ Short channel effects $\cdot \operatorname{In}_{0.53} \mathrm{Ga}_{0.47} \mathrm{As}$

\section{Introduction}

The scaling of conventional bulk Si-MOSFET approaches the fundamental limit due to the increase of off-leakage current and short channel effects [1]. Further performance

Shohiro Sho

shohiro@cas.cmc.osaka-u.ac.jp

1 Computer Assisted Science Division, Cybermedia Center, Osaka University, Toyonaka, Osaka 560-0043, Japan

2 Department of Electronics, Kyoto Institute of Technology, Kyoto, Japan improvements require new channel materials such as $\mathrm{Ge}$ and III-V compound semiconductors [2] and new device structures such as FinFETs [3] and nanowire gate-all-around structures [4]. Performance analysis of single and multigate MOSFETs on high mobility substrates and $\mathrm{Si}$ is an important issue. A number of authors have focused on numerical and theoretical studies of such devices, using self-consistent Poisson/Monte Carlo simulations [5,6], comprehensive semiclassical multisubband Monte Carlo simulations [7], self-consistent solutions of Schrödinger/Poisson equations [6,8,9], a quantum-corrected Monte Carlo simulations [10], and an atomistic Schrödinger/Poisson equations in the non-equilibrium Green's function formalism [11].

This paper describes performance analysis of $\mathrm{Si}, \mathrm{Ge}$, and $\mathrm{In}_{0.53} \mathrm{Ga}_{0.47}$ As n-MOSFETs using a quantum energy transport (QET) model based on Fermi-Dirac statistics and nonparabolicity. The QET model is viewed as one of the hierarchy of the quantum hydrodynamic models [12], which allows simulations of carrier transport including quantum confinement and hot carrier effects [13]. The simulation study focuses on the analysis of quantum confinement and short channel effects. Both bulk and double-gate n-MOSFETs are simulated.

The paper is organized as follows: In Sect. 2, we describe a four-moments QET model based on Fermi-Dirac statistics and nonparabolicity. Section 3 presents numerical simulations of the QET model. The results are further compared with those calculated by quantum drift diffusion (QDD) and classical energy transport (ET) models. The analysis of short channel and quantum confinement effects of $\mathrm{Si}, \mathrm{Ge}$, and $\mathrm{In}_{0.53} \mathrm{Ga}_{0.47} \mathrm{As} \mathrm{n}$-MOSFETs for bulk and double-gate structures is presented. The dependence of short channel effects on channel materials is discussed. Section 4 concludes this paper. 


\section{QET model}

\subsection{4-moments QET model based on Fermi-Dirac statistics}

For the simulations of quantum confinement transport with hot carrier effects, we develop a four-moments QET model in [13]. This model is viewed as one of the hierarchy of the quantum hydrodynamic models [12]. In classical hydrodynamic simulations, a four-moments energy transport model is proposed in [14] for simulations of thin body MOSFETs. In this work, Fermi-Dirac statistics and nonparabolic corrections are further included for the performance analysis of MOSFETs on high mobility substrates. In fact, high mobility materials such as III-V compound semiconductors have strong degeneracy, low density of state, and nonparabolic band structures [15].

Numerical implementation of Fermi-Dirac statistics is discussed in [16] for QDD models and in [17] for QET models. The electron density $n$ is approximated by introducing the band parameter $\omega_{n}$ as

$n=n_{i} \exp \left(\frac{q}{k T_{n}}\left(\varphi+\gamma_{n}+\omega_{n}-\varphi_{n}\right)\right)$,

where $\varphi, \varphi_{n}$, and $T_{n}$ are the electrostatic potential, quasiFermi-level, and electron temperature, respectively. $n_{i}, q$, and $k$ are the intrinsic carrier density, electronic charge, and Boltzmann constant, respectively. The quantum potential $\gamma_{n}$ is described as

$\gamma_{n}=\frac{\hbar^{2}}{6 m q} \frac{1}{\sqrt{n}} \frac{\partial^{2}}{\partial x_{j}^{2}} \sqrt{n}$,

where $m$ and $\hbar$ are the effective mass and Plank constant. The band parameter $\omega_{n}$ is determined as

$\omega_{n}=\frac{k T_{n}}{q}\left(\log \left(\frac{n}{N_{c}}\right)-G_{\frac{1}{2}}\left(\frac{n}{N_{c}}\right)\right)$,

where $N c$ is the density of states in the conduction band, and $G_{\frac{1}{2}}$ is the inverse Fermi function of order $1 / 2$ defined with

$G_{\frac{1}{2}}\left(\frac{2}{\pi} \cdot F_{\frac{1}{2}}^{\alpha}(x)\right)=x$.

The carrier density $n$ including nonparabolic band effects is given by [18]

$n=N_{c} \frac{2}{\pi} F_{\frac{1}{2}}^{\alpha}(\eta)=N_{c} \frac{2}{\pi} \int_{0}^{\infty} \frac{(1+2 \alpha \epsilon) \sqrt{\epsilon(1+\alpha \epsilon)}}{1+\exp (\epsilon-\eta)} d \epsilon$, where $\eta=\left(E_{f}-E_{c}\right) / k T$ is the normalized Fermi level. The parameter $\alpha$ is a coefficient of nonparabolicity that can be calculated as

$\alpha=\frac{1}{\epsilon_{g}}\left(1-\frac{m}{m_{0}}\right)^{2}$,

where $\epsilon_{g}$ is the normalized band gap $\left(=E_{c}-E_{v} / k T\right)$ and $m_{0}$ is the free electron rest mass. The simple analytical approximation of the inverse Fermi function is given in [19] for a weak degenerate case $(\eta<10)$. For high $\eta$, we apply Sommerfeld's approximation to calculate the inverse Fermi function. Both approximations are linearly interpolated.

By employing the expression (1) in the QET model, we obtain the current density

$J_{n}=q \mu_{n}\left(\nabla\left(n \frac{k T_{n}}{q}\right)-n \nabla\left(\varphi+\gamma_{n}+\omega_{n}\right)\right)$,

where $\mu_{n}$ is the electron mobility. From (2), the quantum potential equation is obtained as

$2 b_{n} \nabla^{2} \rho_{n}-\gamma_{n} \rho_{n}=0$,

where $b_{n}=\frac{\hbar^{2}}{12 q m}$. The root-density $\rho_{n}$ is written as $\rho_{n}=$ $\sqrt{n}=\sqrt{n_{i}} \exp \left(u_{n}\right)$ by a variable $u_{n}=\frac{q}{k T_{n}}\left(\frac{\left(\varphi+\gamma_{n}+\omega_{n}-\varphi_{n}\right)}{2}\right)$ in (1). As shown in [16], under Fermi-Dirac statistics, (8) is replaced by the equivalent form

$b_{n} \nabla \cdot\left(\rho_{n} \nabla u_{n}\right)-\frac{k T_{n}}{q} \rho_{n} u_{n}=-\frac{\rho_{n}}{2}\left(\varphi+\omega_{n}-\varphi_{n}\right)$.

If the variable $u_{n}$ is uniformly bounded, the electron density is maintained to be positive. This approach provides a numerical advantage for developing an iterative solution method.

For electrons, the four-moments QET model based on Fermi-Dirac statistics is described as follows:

$$
\begin{aligned}
& \epsilon \Delta \varphi=q\left(n-p-C_{i m p}\right), \\
& \frac{1}{q} \operatorname{div} J_{n}=0, \\
& J_{n}=q \mu_{n}\left(\nabla\left(n \frac{k T_{n}}{q}\right)-n \nabla\left(\varphi+\omega_{n}+\gamma_{n}\right)\right), \\
& b_{n} \nabla \cdot\left(\rho_{n} \nabla u_{n}\right)-\frac{k T_{n}}{q} \rho_{n} u_{n}=-\frac{\rho_{n}}{2}\left(\varphi+\omega_{n}-\varphi_{n}\right), \\
& \nabla \cdot S_{n}=-J_{n} \cdot \nabla \varphi-\frac{3}{2} k n \frac{T_{n}-T_{L}}{\tau_{\epsilon}}, \\
& S_{n}=-\frac{\mu_{s}}{\mu_{n}}\left(\frac{5}{2} \frac{k T_{n}}{q}-\frac{\hbar^{2}}{24 m q} \Delta \log n-\gamma_{n}\right) J_{n} \\
& \quad-\frac{\mu_{s}}{\mu_{n}} \frac{5}{2}\left(\frac{k}{q}\right)^{2} q \mu_{n} n T_{n} \nabla T_{n},
\end{aligned}
$$


Table 1 Parameters for the low-field mobility model

\begin{tabular}{llll}
\hline Material & $\mathrm{Si}[25]$ & $\mathrm{Ge}[25]$ & $\mathrm{In}_{0.53} \mathrm{Ga}_{0.47} \mathrm{As}[26]$ \\
\hline$\mu_{L}\left(\mathrm{~cm}^{2} / V s\right)$ & 1400 & 3900 & 14000 \\
$\mu_{\text {min }}\left(\mathrm{cm}^{2} / V s\right)$ & 80 & 850 & 300 \\
$C_{\text {ref }}$ & $1.12 \mathrm{e} 17$ & $2.6 \mathrm{e} 17$ & $1.3 \mathrm{e} 17$ \\
$\alpha_{L F}$ & 0.72 & 0.56 & 0.48 \\
\hline
\end{tabular}

where $p, \epsilon$ and $C_{i m p}$ are the hole density, the permittivity of semiconductor, and the ionized impurity density, respectively. $T_{L}$ and $\tau_{\epsilon}$ are the lattice temperature and energy relaxation time. The ratio $\mu_{n} / \mu_{s}$ selected here is 0.8 [20]. For holes, similar expressions are obtained.

\subsection{Mobility model}

For the energy dependence of the mobility, we apply the model of the Baccarani et al. [21],

$\frac{\mu_{n}\left(T_{n}\right)}{\mu_{L F}}=\frac{T_{L}}{T_{n}}$.

In the homogeneous case, this model is equivalent to the Hänsch mobility model [20]

$$
\frac{\mu_{n}\left(T_{n}\right)}{\mu_{L F}}=\left(1+\frac{3}{2} \frac{\mu_{L F} k}{q \tau_{\epsilon} v_{s}^{2}}\left(T_{n}-T_{L}\right)\right)^{-1},
$$

where $v_{s}$ is the saturation velocity. As mentioned in [22,23], the Hänsch mobility model is consistent with the high-field mobility model

$$
\frac{\mu_{n}(E)}{\mu_{L F}}=\frac{1}{\xi+\left((1-\xi)^{\beta}+\left(\frac{\mu_{L F} \cdot E}{v_{s a t}}\right)^{\beta}\right)^{\frac{1}{\beta}}}
$$

with the parameters $\xi=1 / 2$ and $\beta=2$.

To account for the mobility reduction due to the ionized impurity scattering, we use the formula of Caughey and Thomas [24] for the low-field mobility $\mu_{L F}$ in this work:

$\mu_{L F}=\mu_{\min }+\frac{\mu_{L}-\mu_{\min }}{1+\left(\frac{C}{C_{r e f}}\right)^{\alpha_{L F}}}$.

The model parameter values $[25,26]$ are summarized in Table 1. In this work, numerical simulations are performed by using the Baccarani's mobility model (16) for the QET model and the high-field mobility model (18) for the QDD model, respectively. The effects of interface traps and surface roughness scattering are not included in this work.

\section{Simulation results}

\subsection{Device structures}

The schematic views of simulated devices are shown in Fig. 1. $\mathrm{Si}, \mathrm{Ge}$, and $\mathrm{In}_{0.53} \mathrm{Ga}_{0.47}$ As n-MOSFETs with high-k/metal gates are examined. Selected material parameters are listed in Table 2. The relative dielectric permittivity considered here is 22 , and the value is known as " $\mathrm{HfO}_{2}$ ". The equivalent oxide thickness (EOT) is $0.6 \mathrm{~nm}$. The threshold of all devices is obtained by the adjustment of the gate work function, which is selected for each semiconductor material to meet a common threshold voltage of $0.2 \mathrm{~V}$. The threshold voltage is defined as the gate voltage when the drain current is $10 \mu \mathrm{A} / \mu \mathrm{m}$. The channel length of simulated devices is varied from 35 to $16 \mathrm{~nm}$. The S/D doping is $N_{S D}=$
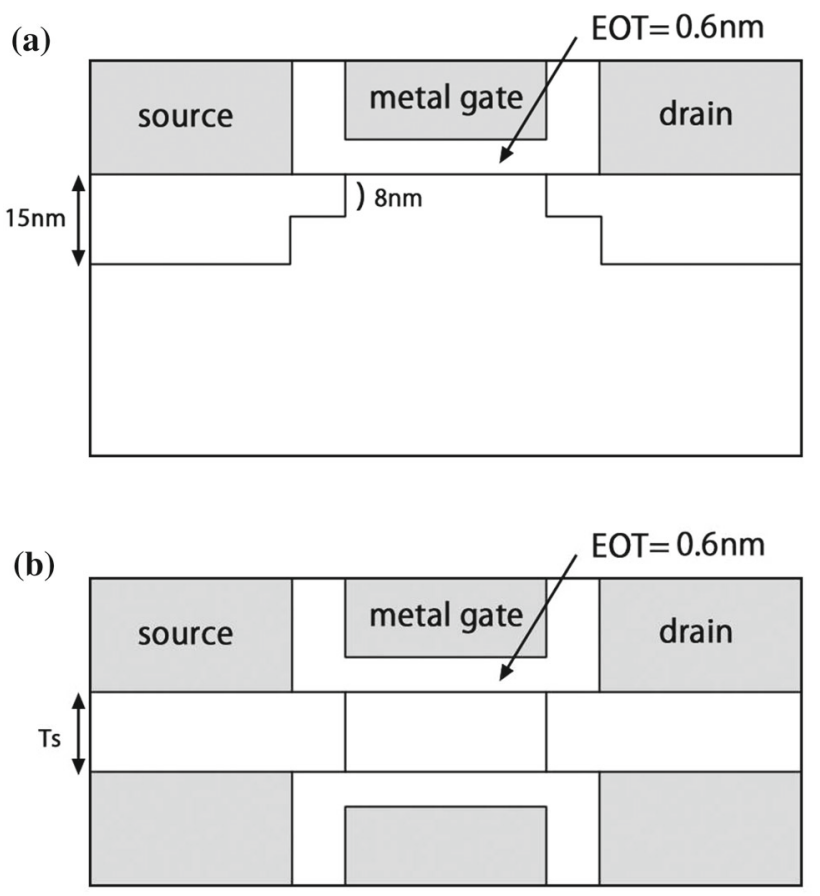

Fig. 1 Schematic views of simulated (a) Bulk and (b) DG MOSFETs. The channel length is varied from 35 to $16 \mathrm{~nm}$

Table 2 Selected semiconductor material parameters

\begin{tabular}{llll}
\hline Semiconductor & $\mathrm{Si}$ & $\mathrm{Ge}$ & $\mathrm{In}_{0.53} \mathrm{Ga}_{0.47} \mathrm{As}$ \\
\hline$E_{G}(\mathrm{eV})$ & $1.12[6]$ & $0.66[6]$ & $0.73[6]$ \\
$\epsilon_{R}\left(\epsilon_{0}\right)$ & $11.7[6]$ & $16.0[6]$ & $14.0[6]$ \\
$m_{e f f}\left(m_{0}\right)$ & $0.26[29]$ & $0.12[29]$ & $0.048[6]$ \\
$n_{i}\left(\mathrm{~cm}^{-3}\right)$ & $1.08 \mathrm{e} 10[29]$ & $1.64 \mathrm{e} 13[29]$ & $9.0 \mathrm{e} 11[28]$ \\
$v_{s a t}(\mathrm{~cm} / \mathrm{s})$ & $1.0 \mathrm{e} 7[27]$ & $0.7 \mathrm{e} 7[27]$ & $0.75 \mathrm{e} 7[27]$ \\
$N_{S D}\left(\mathrm{~cm}^{-3}\right)$ & $1.0 \mathrm{e} 20$ & $1.0 \mathrm{e} 20$ & $2.0 \mathrm{e} 19[2]$ \\
$N_{c}\left(\mathrm{~cm}^{-3}\right)$ & $2.86 \mathrm{e} 19$ & $1.05 \mathrm{e} 19$ & $2.64 \mathrm{e} 17$ \\
\hline
\end{tabular}



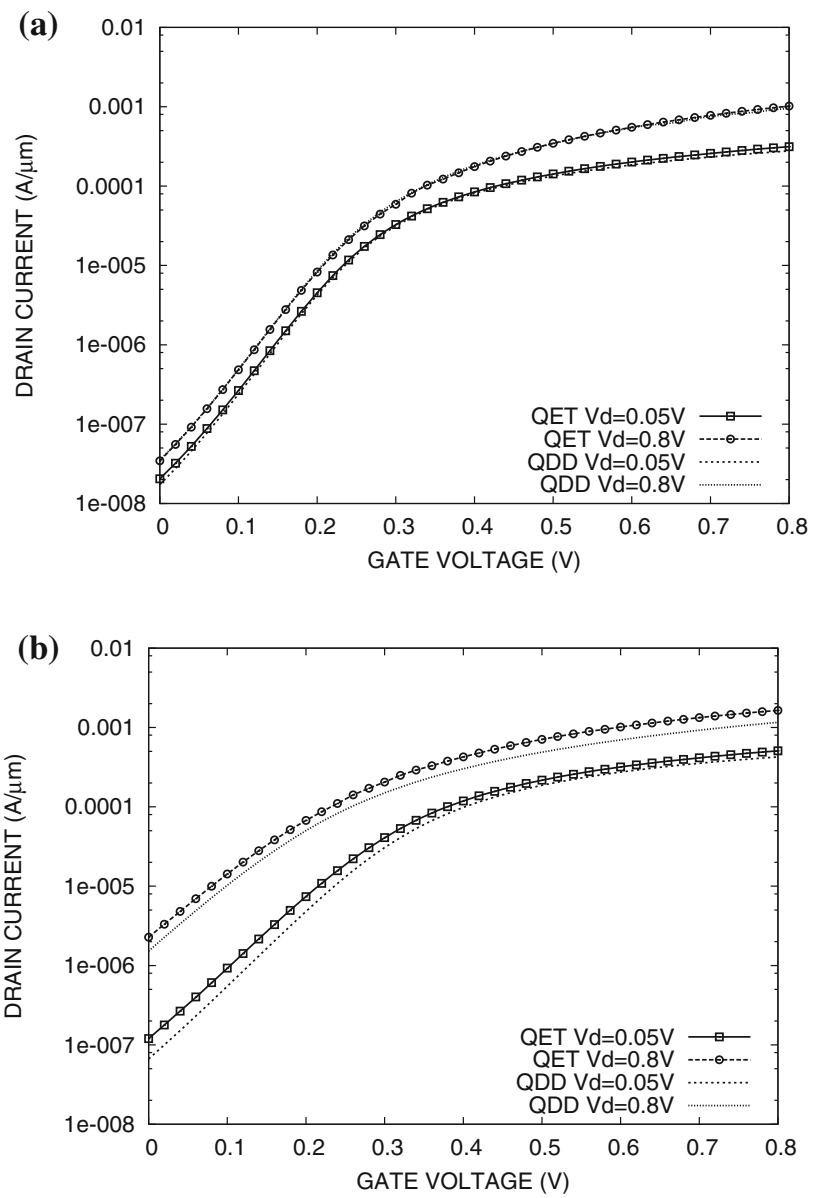

Fig. 2 Comparison of the $I_{d}-V_{G}$ characteristics of $\mathrm{Si}$ bulk nMOSFETs between QET and QDD models. $\mathbf{a} \mathrm{Lg}=50 \mathrm{~nm}, \mathbf{b} \mathrm{Lg}=20 \mathrm{~nm}$

$1.0 \times 10^{20} \mathrm{~cm}^{-3}$ for Si and Ge n-MOSFETs. Since activated donor concentrations larger than $2.0 \times 10^{19} \mathrm{~cm}^{-3}$ cannot be obtained in $\operatorname{In}_{0.53} \mathrm{Ga}_{0.47}$ As n-MOSFETs [2], we set up $N_{S D}=2.0 \times 10^{19} \mathrm{~cm}^{-3}$ for an $\operatorname{In}_{0.53} \mathrm{Ga}_{0.47}$ As n-MOSFET. We further assume channel dopings of $2.0 \times 10^{18} \mathrm{~cm}^{-3}$ for bulk n-MOSFETs, and $1.0 \times 10^{15} \mathrm{~cm}^{-3}$ for double-gate $\mathrm{n}$ MOSFETs.

\subsection{QET simulations}

Figure 2a and $\mathrm{b}$ demonstrates the $\mathrm{I}_{D^{-}} \mathrm{V}_{G}$ characteristics of 50 and $20 \mathrm{~nm}$ Si bulk n-MOSFETs at $V_{d}=0.05 \mathrm{~V}$ and $0.8 \mathrm{~V}$, which are calculated by the QET and QDD models. The same work function is used for both models. In a long channel device, the $\mathrm{I}_{D}-\mathrm{V}_{G}$ characteristics calculated by two models are almost identical, as shown in Fig. 2a. For the ultra-short channel device, two models exhibit the different results of $\mathrm{I}_{D}-\mathrm{V}_{G}$ characteristics due to the non-local transport effects and the reduction of the quantum confinement effects. The QET model provides higher drain current. The subthreshold

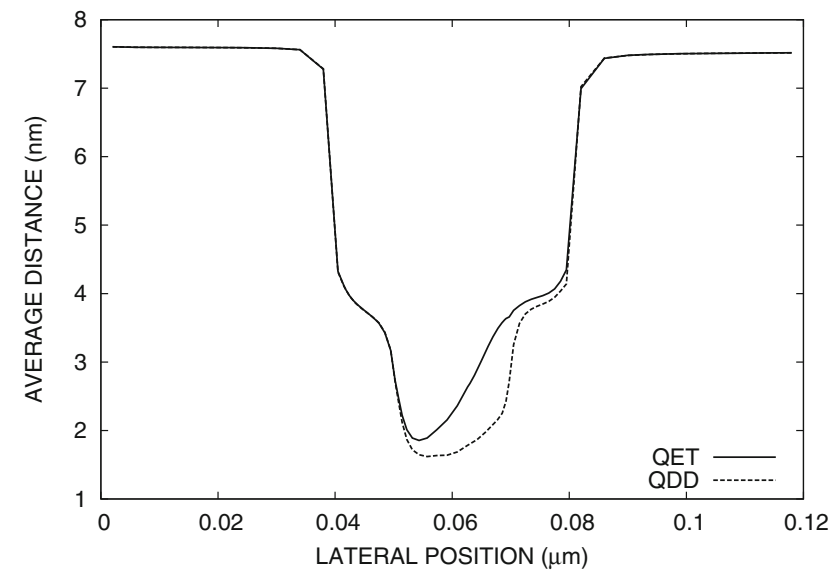

Fig. 3 Comparison of the average distance of carriers from interface for a $20 \mathrm{~nm}$ Si bulk n-MOSFET between QET and QDD models
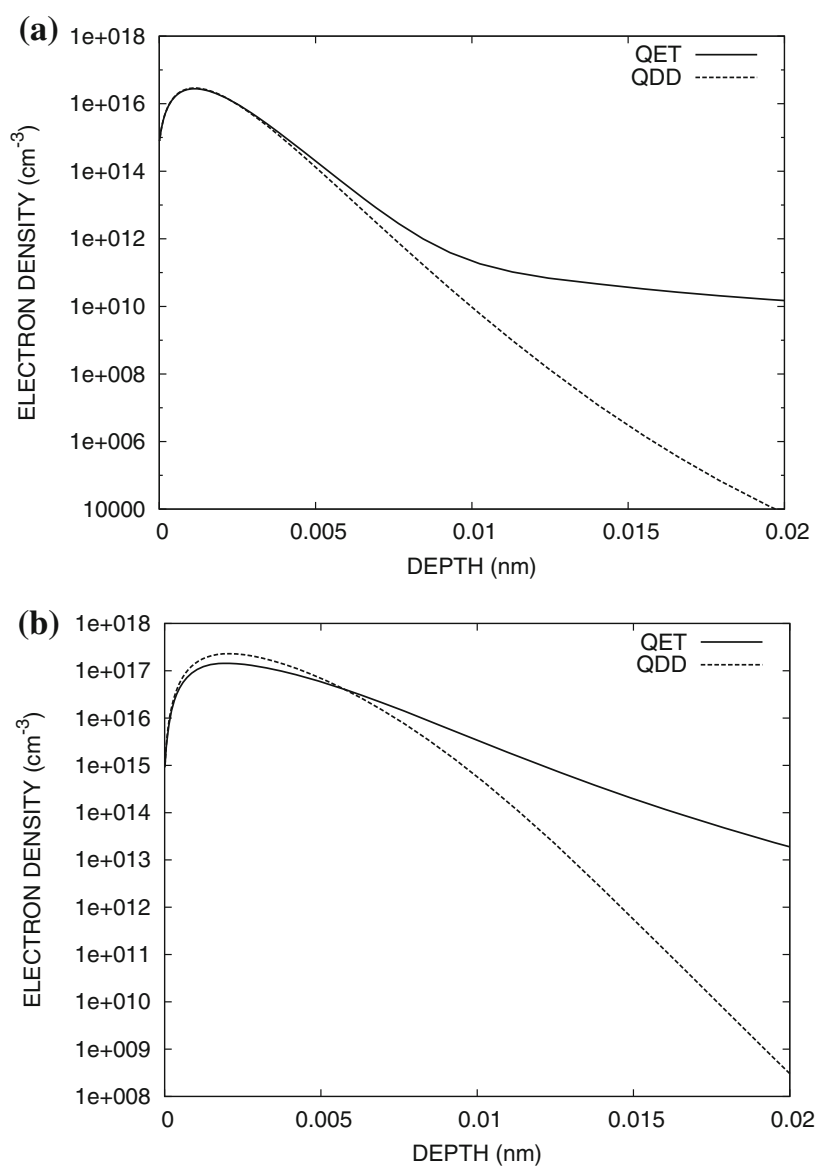

Fig. 4 Electron density distributions perpendicular to the interface for Si bulk n-MOSFETs calculated by QET and QDD models at the center of the channel. $\mathrm{Vg}=0.1 \mathrm{~V}, \mathrm{Vd}=0.8 \mathrm{~V} . \mathbf{a} \mathrm{Lg}=50 \mathrm{~nm}, \mathbf{b ~ L g}=20 \mathrm{~nm}$

slope (SS) calculated by the QET model is increased from $107 \mathrm{mV} / \mathrm{dec}$ to $114 \mathrm{mV} / \mathrm{dec}$. Figure 3 shows the average distance of electrons from the interface calculated by the QET and QDD models. The simulations are done at $V_{g}=0.8 \mathrm{~V}$ and $V_{d}=0.8 \mathrm{~V}$. The hot electron effects result in the spread 

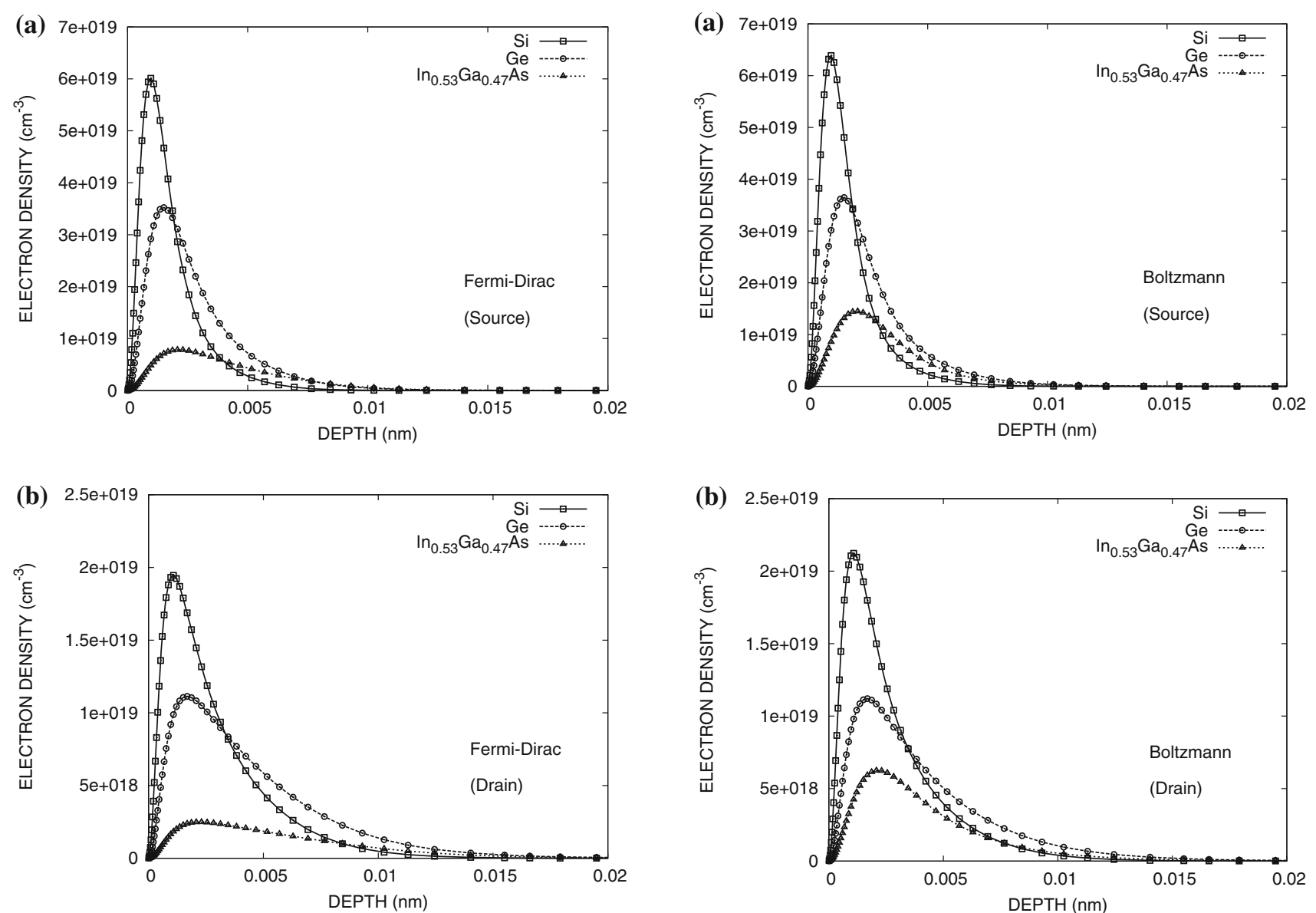

Fig. 5 Electron density distributions perpendicular to the interface for $20 \mathrm{~nm} \mathrm{Si}, \mathrm{Ge}$, and $\mathrm{In}_{0.53} \mathrm{Ga}_{0.47}$ As bulk n-MOSFETs calculated by the QET model based on Fermi-Dirac statistics. a at the source end of the channel, $\mathbf{b}$ at the drain end of the channel. $V_{g}=0.8 \mathrm{~V}, V_{d}=0.8 \mathrm{~V}$

of electrons toward the bulk in the channel and hence in ultrashort channel devices, a significant difference between two models is induced. The results clearly indicate that the quantum confinement effect in the ultra-short channel is reduced by the enhanced diffusion due to the high electron temperature. Figure $4 \mathrm{a}$ and $\mathrm{b}$ shows the electron density distributions calculated by the QET and QDD models for long and short channel devices. The results are plotted at the center of the channel. In the long channel device, the electron density distributions calculated by two models are almost identical at the surface. For the ultra-short channel device, due to the hot carrier effects, the electron density distribution calculated by the QET model is spread towards the bulk. This result in the reduction of charge control calculated by the QET model.

\subsection{Quantum confinement effects}

The dependence of quantum confinement effects on channel materials is investigated in Figs. 5 and 6. The results under Fermi-Dirac statistics and Boltzmann statistics are

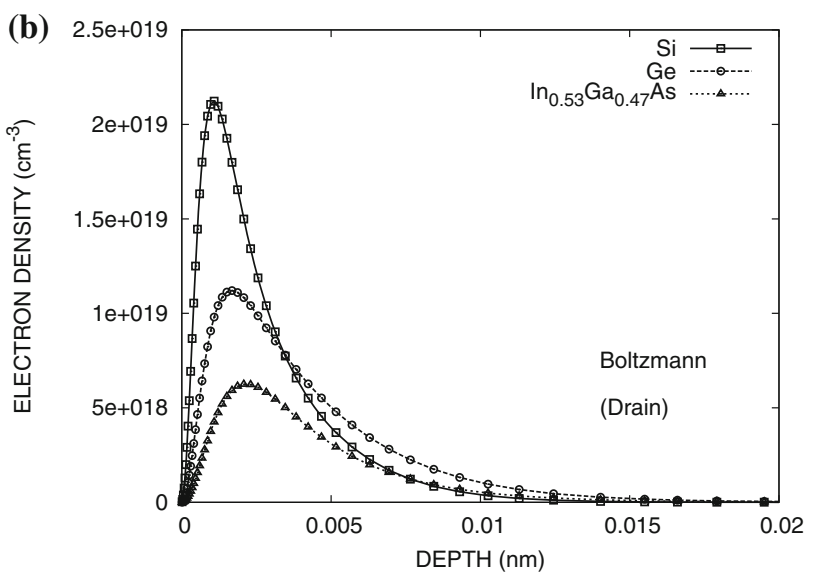

Fig. 6 Electron density distributions perpendicular to the interface for $20 \mathrm{~nm} \mathrm{Si}, \mathrm{Ge}$, and $\mathrm{In}_{0.53} \mathrm{Ga}_{0.47} \mathrm{As}$ bulk n-MOSFETs calculated by the QET model based on Boltzmann statistics. $\mathbf{a}$ at the source end of the channel, $\mathbf{b}$ at the drain end of the channel. $V_{g}=0.8 \mathrm{~V}, V_{d}=0.8 \mathrm{~V}$

compared. The electron density distributions for $20 \mathrm{~nm} \mathrm{Si}$, Ge and $\operatorname{In}_{0.53} \mathrm{Ga}_{0.47}$ As bulk n-MOSFETs are plotted at the source and drain ends of the channel. The devices are simulated at $V_{g}=0.8 \mathrm{~V}$ and $V_{d}=0.8 \mathrm{~V}$. As shown in Fig. $5 \mathrm{a}$, the inversion layer electrons in $\mathrm{Ge}$ and $\mathrm{In}_{0.53} \mathrm{Ga}_{0.47} \mathrm{As}$ n-MOSFETs spread into the bulk at the source end of the channel due to the low effective mass and high permittivity. Figure $5 \mathrm{~b}$ reveals that in all devices the quantum confinement effect is further reduced by the enhanced diffusion due to the high electron temperature. These properties degrade the short channel effects of $\mathrm{Ge}$ and $\mathrm{In}_{0.53} \mathrm{Ga}_{0.47}$ As devices when compared with $\mathrm{Si}$ devices, as discussed later. Since $N_{c}\left(=2.64 \times 10^{17} \mathrm{~cm}^{-3}\right)$ of $\operatorname{In}_{0.53} \mathrm{Ga}_{0.47}$ As is low, the inversion layer electrons in the $\operatorname{In}_{0.53} \mathrm{Ga}_{0.47} \mathrm{As}$ n-MOSFET are further decreased due to the high degeneracy material.

In Fig. 7a and b, we compare the electron density distributions calculated by the QET model based on Fermi-Dirac statistics for $20 \mathrm{~nm} \mathrm{Si}, \mathrm{Ge}$, and $\mathrm{In}_{0.53} \mathrm{Ga}_{0.47}$ As double-gate nMOSFETs, respectively. The double-gate structures having a film thickness of $8 \mathrm{~nm}$ are simulated. The results are plot- 

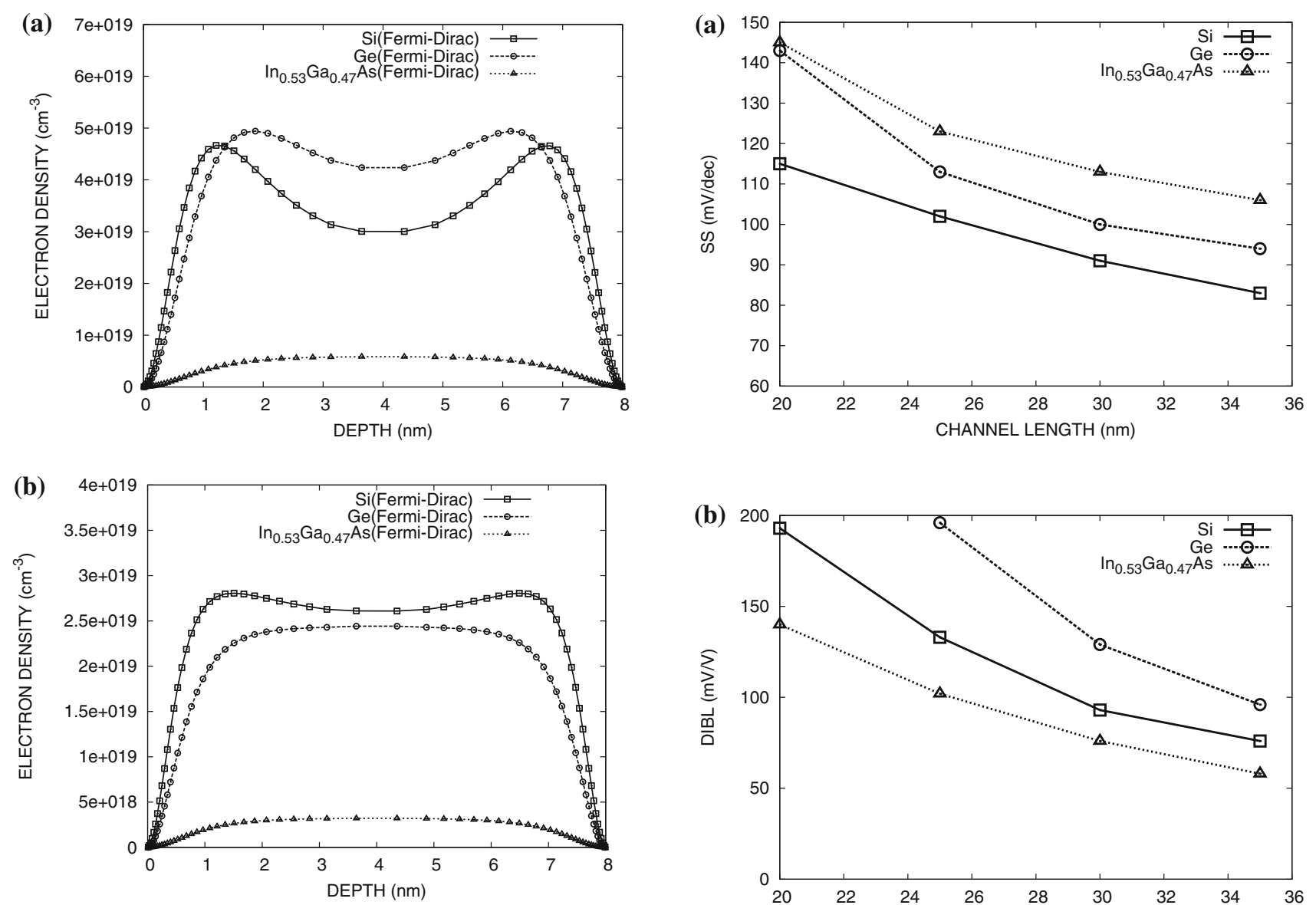

Fig. 7 Electron density distributions perpendicular to the interface for $20 \mathrm{~nm} \mathrm{Si}, \mathrm{Ge}$, and $\mathrm{In}_{0.53} \mathrm{Ga}_{0.47}$ As doube gate n-MOSFETs calculated by the QET model based on Fermi-Dirac statistics. $\mathbf{a}$ at the source end of the channel, $\mathbf{b}$ at the drain end of the channel. $V_{g}=0.8 \mathrm{~V}, V_{d}=0.8$

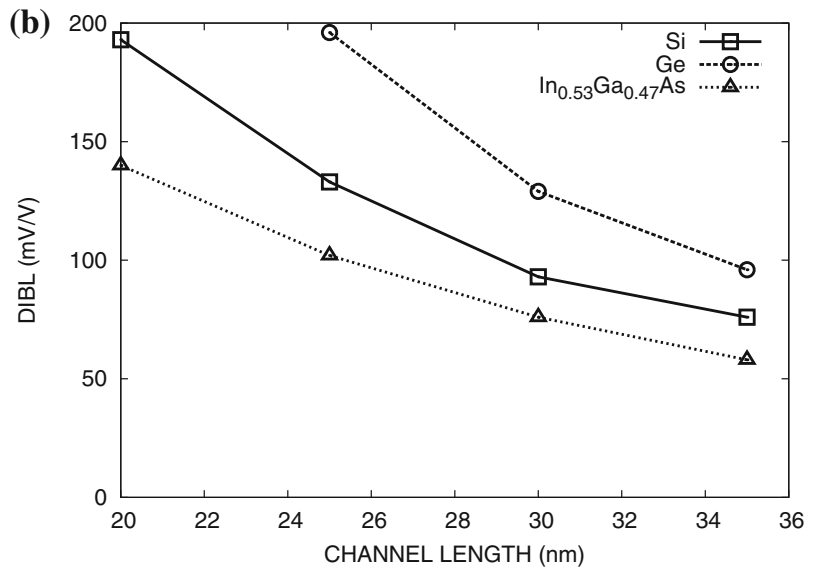

Fig. 8 a Subthreshold slope at $V_{d}=0.05 \mathrm{~V}$ versus channel length of $\mathrm{Si}, \mathrm{Ge}$, and $\mathrm{In}_{0.53} \mathrm{Ga}_{0.47}$ As bulk n-MOSFETs. b Drain-induced barrier lowering versus channel length of bulk $\mathrm{Si}, \mathrm{Ge}$, and $\mathrm{In}_{0.53} \mathrm{Ga}_{0.47} \mathrm{As} \mathrm{n}$ MOSFETs

ted at the source and drain ends of the channel. The devices are simulated at $V_{g}=0.8 \mathrm{~V}$ and $V_{d}=0.8 \mathrm{~V}$. The inversion layer electrons in $\mathrm{Ge}$ and $\mathrm{In}_{0.53} \mathrm{Ga}_{0.47} \mathrm{As}$ n-MOSFETs spread into the center of the channel due to the low effective mass and high permittivity. In analogy to the results of bulk n-MOSFETs, the single inversion layer electrons in the $\mathrm{In}_{0.53} \mathrm{Ga}_{0.47} \mathrm{As} \mathrm{n}$-MOSFET are further decreased due to the high degeneracy material. In Fig. 7a, Si and Ge n-MOSFETs exhibit two inversion layers at the source end of the channel. In the film thickness of $8 \mathrm{~nm}$, Ge n-MOSFETs form a single inversion layer due to the hot electron effects at the drain end of the channel as shown in Fig. $7 b$.

\subsection{Short channel effects}

Figure $8 \mathrm{a}$ and $\mathrm{b}$ indicates the SS and DIBL as a function of the channel length for $\mathrm{Si}, \mathrm{Ge}$, and $\mathrm{In}_{0.53} \mathrm{Ga}_{0.47}$ As bulk nMOSFETs. The simulations are performed by using the QET model based on Fermi-Dirac statistics. As seen in Fig. 8a, the SS of $\mathrm{Ge}$ and $\mathrm{In}_{0.53} \mathrm{Ga}_{0.47} \mathrm{As}$-MOSFETs is larger than that

of the Si n-MOSFET due to smaller electron effective mass and higher permittivity. It is shown in Fig. $8 \mathrm{~b}$ that the DIBL of $\mathrm{In}_{0.53} \mathrm{Ga}_{0.47} \mathrm{As}$ n-MOSFET is suppressed because of the low S/D doping concentration. Fig. 9a and b shows the dependence of SS and DIBL on the channel length for $\mathrm{Si}, \mathrm{Ge}$, and $\mathrm{In}_{0.53} \mathrm{Ga}_{0.47}$ As double-gate n-MOSFETs. The short channel effects are suppressed in the multi-gate structure. A different improvement between SS and DIBL is seen. In all devices, the DIBL effect is reduced in double-gate n-MOSFETs. In the $\mathrm{In}_{0.53} \mathrm{Ga}_{0.47}$ As $\mathrm{n}$-MOSFET, the DIBL effect is significantly reduced due to the low S/D doping concentration. This is because the thin film suppresses an extension of drain electric field into the channel. The SS improvement depends on the channel material even in double gate structure. The SS of Ge and $\mathrm{In}_{0.53} \mathrm{Ga}_{0.47} \mathrm{As}$ n-MOSFETs are larger than that of Si n-MOSFET due to the low effective mass and high permittivity as well as the results of bulk n-MOSFETs. Figure 10 shows the threshold voltage roll-off of $\mathrm{Si}, \mathrm{Ge}$, and $\mathrm{In}_{0.53} \mathrm{Ga}_{0.47}$ As n-MOSFETs at $V_{d}=0.5 \mathrm{~V}$. The results of 

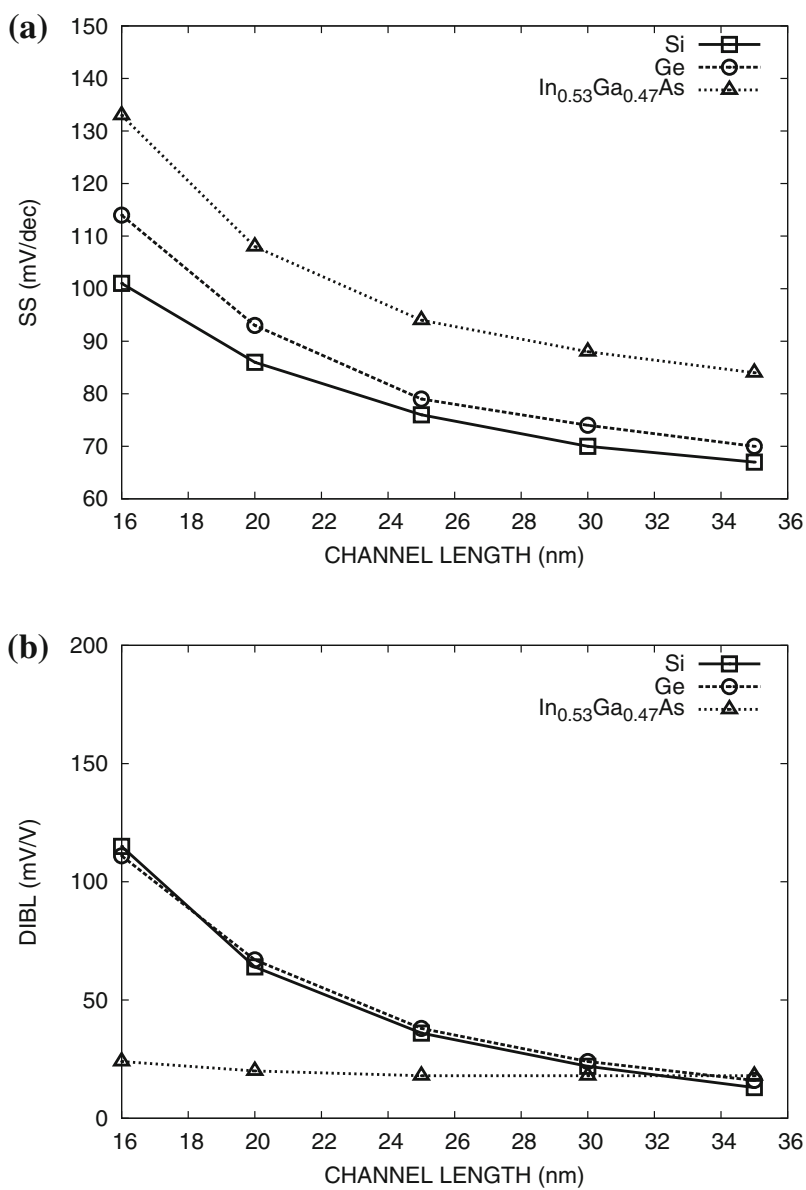

Fig. 9 a Subthreshold slope at $V_{d}=0.05 \mathrm{~V}$ versus channel length of $\mathrm{Si}, \mathrm{Ge}$, and $\mathrm{In}_{0.53} \mathrm{Ga}_{0.47} \mathrm{As}$ double-gate n-MOSFETs. b Drain-induced barrier lowering versus channel length of $\mathrm{Si}, \mathrm{Ge}$, and $\mathrm{In}_{0.53} \mathrm{Ga}_{0.47} \mathrm{As}$ double-gate n-MOSFETs

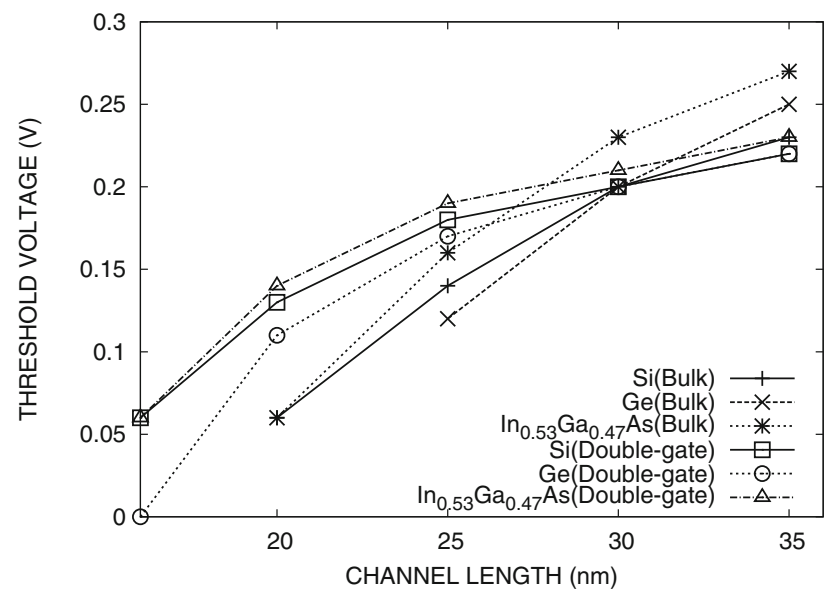

Fig. 10 Threshold voltage roll-off at $V_{d}=0.5 \mathrm{~V}$ versus channel length of $\mathrm{Si}, \mathrm{Ge}, \mathrm{In}_{0.53} \mathrm{Ga}_{0.47}$ As bulk and double-gate n-MOSFETs.

both bulk and double-gate n-MOSFETs are shown. The $V_{T}$ roll-off of the $\operatorname{In}_{0.53} \mathrm{Ga}_{0.47}$ As n-MOSFET is almost the same as that of Si n-MOSFET in the double gate structure because of the low S/D doping concentration. The Ge n-MOSFET shows the worst short channel effects.

\section{Conclusion}

The quantum confinement and short channel effects of $\mathrm{Si}, \mathrm{Ge}$, and $\mathrm{In}_{0.53} \mathrm{Ga}_{0.47} \mathrm{As} \mathrm{n}$-MOSFETs have been evaluated using a 4-moments QET model based on Fermi-Dirac statistics and nonparabolicity. The dependence of quantum confinement effects on channel materials has been clarified. The charge control by the gate is reduced in $\mathrm{Ge}$ and $\mathrm{In}_{0.53} \mathrm{Ga}_{0.47} \mathrm{As}$ n-MOSFETs due to the low effective mass and high permittivity. This results in the degradation of short channel effects. The double-gate structure is effective in the suppression of DIBL for all channel materials. The SS degradation depends on channel materials even in double-gate structure.

Open Access This article is distributed under the terms of the Creative Commons Attribution 4.0 International License (http://creativecomm ons.org/licenses/by/4.0/), which permits unrestricted use, distribution, and reproduction in any medium, provided you give appropriate credit to the original author(s) and the source, provide a link to the Creative Commons license, and indicate if changes were made.

\section{References}

1. Bohr, M.: The evolution of scaling from the homogeneous era to the heterogeneous era. In: Proceedings of IEDM, pp. 1.1.1-1.1.6 (2011)

2. Fischetti, M.V., Laux, S.E.: Monte Carlo simulation of transport in technologically significant semiconductors of the diamond and Zinc-Blende structures-Part II: submicrometer MOSFET's. IEEE Trans. Electron Devices 38, 650-660 (1991)

3. Hisamoto, D., Lee, W.-C., Kedzierski, J., Takeuchi, H., Kuo, C., Anderson, E., King, T.-J., Bokor, J., Hu, C.: FinFET-A self-aligned double-gate MOSFET scalable to $20 \mathrm{~nm}$. IEEE Trans. Electron Devices 47, 2320-2325 (2000)

4. Singh, N., Agrwal, A., Bera, L.K., Liow, T.Y., Yang, R., Rustagi, S.C., Tung, C.H., Kumer, R., Lo, G.Q., Balasubramanian, N., Kwong, D.L.: High-performance fully depleted silicon nanowire (diameter $\leq 5 \mathrm{~nm}$ ) gate-all-around CMOS devices. IEEE Electron Device Lett. 27, 383-386 (2006)

5. Fischetti, M.V., O'Regan, T.P.: Theoretical study of some physical aspects of electronic transport in nMOSFETs at the 10-nm gatelength. IEEE Trans. Electron Devices 54, 2116-2136 (2007)

6. Laux, S.E.: A simulation study of the switching times of 22- and 17-nm gate-length SOI nFETs on high mobility substrates and Si. IEEE Trans. Electron Devices 54, 2304-2320 (2007)

7. Lizzit, D., Esseni, D., Palestri, P., Osgnach, P., Selmi, L.: Performance benchmarking and effective channel length for nanoscale InAs, $\mathrm{In}_{0.53} \mathrm{Ga}_{0.47} \mathrm{As}$, and sSi n-MOSFETs. IEEE Trans. Electron Devices 61, 2027-2034 (2014)

8. Park, S.H., Liu, Y., Kharche, N., Jelodar, M.S., Klimeck, G., Lundstrom, M.S., Luisier, M.: Performance comparisons of III-V and strained-Si in planar FETs and nonplanar FinFETs at ultrashort gate length(12nm). IEEE Trans. Electron Devices 59, 2107-2114 (2012) 
9. Moreau, M., Munteanu, D., Autran, J.L.: Simulation study of shortchannel effects and quantum confinement in double-gate FinFET devices with high-mobility materials. Microelectron. Eng. 88, 366369 (2011)

10. Mori, T., Asuma, Y., Tsuchiya, H., Miyoshi, T.: Comparative study on drive current of III-V semiconductor, Ge and $\mathrm{Si}$ channel nMOSFETs based on quantum-corrected Monte Carlo simulation. IEEE Trans. Nanotechnol. 7, 237-241 (2008)

11. Luisier, M.: Performance comparison of GaSb, strained-Si, and InGaAs double-gate ultra-thin-body n-FETs. IEEE Electron Devices 32, 1686-1688 (2011)

12. Gardner, C.L.: The quantum hydrodynamic model for semiconductor devices. SIAM J. Appl. Math. 54, 409-427 (1994)

13. Sho, S., Odanaka, S.: A quantum energy transport model for semiconductor device simulation. J. Comput. Phys. 235, 486-496 (2013)

14. Gritsch, M., Kosina, H., Grasser, T., Selberherr, S.: Revision of the standard hydrodynamic transport model for SOI simulation. IEEE Trans. Electron Devices 49, 1814-1820 (2002)

15. O'Regan, T.P., Hurley, P.K., Soree, B., Fischetti, M.V.: Modeling the capacitance-voltage response of $\mathrm{In}_{0.53} \mathrm{Ga}_{0.47}$ As metal-oxidesemiconductor structures: Charge quantization and nonparabolic corrections. Appl. Phys. Lett. 96, 213514 (2010)

16. Odanaka, S.: Multidimensional discretization of the stationary quantum drift-diffusion model for ultrasmall MOSFET structures. IEEE Trans. CAD ICAS 23, 837-842 (2004)

17. Sho, S., Odanaka, S., Hiroki, A.: A Fermi-Dirac statistics based quantum energy transport model for high mobility MOSFETs. JASSE 2, 153-170 (2015)

18. Bebb, H.B., Ratliff, C.R.: Numerical tabulation of integrals of Fermi functions using $\mathbf{k} \cdot \mathbf{p}$ density of states. J. Appl. Phys. 42, 3189-3194(1971)
19. Altschul, V., Finkman, E.: Simple approximation for Fermi energy in nonparabolic semiconductors. Appl. Phys. Lett. 58, 942-944 (1991)

20. Grasser, T., Tang, T.-W., Kosina, H., Selberherr, S.: A review of hydrodynamic and energy-transport models for semiconductor device simulation. IEEE Proc. 91(2), 251-274 (2003)

21. Baccarani, G., Wordeman, M.R.: An investigation of steady-state velocity overshoot in silicon. Solid-State Elec. 28, 407-416 (1985)

22. Grasser, T., Kosina, H., Selberherr, S.: Consistent comparison of drift diffusion and hydrodynamic device simulations. In: Proceedings of SISPAD, pp. 151-154 (1999)

23. Hänsch, W., Miura-Mattausch, M.: The hot-electron problem in small semiconductor devices. J. Appl. Phys. 60, 650-656 (1986)

24. Caughey, D.M., Thomas, R.E.: Carrier mobilities in silicon empirically related to doping and field. Proc. IEEE 52, 2192-2193 (1967)

25. Selberherr, S.: Analysis and Simulation of Semiconductor Devices. Springer, Wien (1984)

26. Sotoodeh, M., Khalid, A.H., Rezazadeh, A.A.: Empirical low-field mobility model for III-V compounds applicable in device simulation codes. J. Appl. Phys. 87, 2890-2900 (2000)

27. Quey, R., Moglestue, C., Palankovski, V., Selberherr, S.: A temperature dependent model for the saturation velocity in semiconductor materials. Mater. Sci. Semicond. Process. 3, 149-155 (2000)

28. Carmody, C., Tan, H.H., Jagadish, C., Gaarder, A.: Ion-implanted In0.53Ga0.47As for ultrafast optoelectronic applications. Appl. Phys. Lett. 82, 3913-3915 (2003)

29. Anderson, B.L., Anderson, R.L.: Fundamentals of Semiconductor Devices. In: Paulson, C (ed.) McGraw-Hill, New York (2005) 